\title{
Supporting Information: Message passing neural networks for partial charge assignment to metal-organic frameworks
}

\author{
Ali Raza, ${ }^{\dagger}$ Arni Sturluson, ${ }^{\ddagger}$ Cory M. Simon, ${ }^{*}, \ddagger$ and Xiaoli Fern ${ }^{*, \dagger}$ \\ $\dagger$ School of Electrical Engineering and Computer Science. Oregon State University. \\ $\ddagger$ School of Chemical, Biological, and Environmental Engineering. Oregon State University. \\ E-mail: Cory.Simon@oregonstate.edu; xfern@eecs.oregonstate.edu \\ S1 The Prevalence of Elements in the Training, Test- \\ ing, and Validation Set
}

Fig. S1 shows the prevalence of elements in the DFT-derived DDEC charge-assigned MOFs of Nazarian et al. ${ }^{1}$ at both the node (blue) and graph (red) level. The elements $\{\mathrm{Se}, \mathrm{Hf}, \mathrm{Cs}$, $\mathrm{Pu}, \mathrm{Ir}\}$ are contained in only one MOF (each). We elected to keep the MOF structures with these rare elements in our training set, as we can learn about charges on other atoms in these MOFs; however, we refrain from making predictions about the charges on these elements during deployment due to insufficient training data for these elements, which prevents us from having confidence in the predicted charges on these elements. 


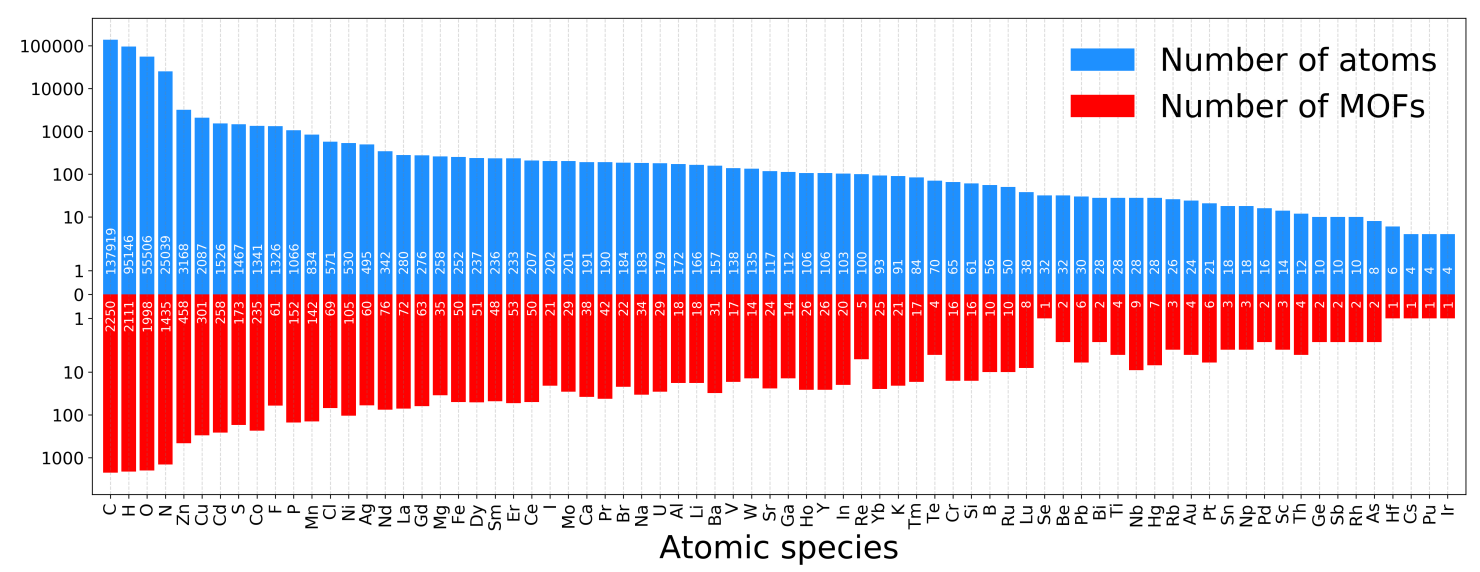

Figure S1: The elemental composition of the DDEC charge-assigned MOFs by Nazarian et al., ${ }^{1}$ composed of 2266 MOFs containing 334103 atoms. Red bars show the number of MOFs containing that specific element. Blue bars show the total number of atoms of that specific element. There are 74 unique elements contained in the MOFs.

\section{S2 Modification of Covalent Radii for Graph Creation}

To guarantee a connected graph when converting the MOF crystal structures ${ }^{1}$ stored in a .cif file to a graph $G$, we made several modifications to the covalent radii $^{2}$ of 10 metal atoms. We based these adjustments on the MOFs below (reported as CSD Refcodes), where our bonding algorithm gave a disconnected graph. The changes made are:

Cd: The covalent radius of $\mathrm{Cd}$ was changed from $1.44 \AA$ to $1.58 \AA$, in order for Cd to bond to neighboring $\mathrm{O}$ and $\mathrm{N}$ atoms in HOWQEQ.

Ag: The covalent radius of $\mathrm{Ag}$ was changed from $1.45 \AA$ to $1.65 \AA$, in order for $\mathrm{Ag}$ to bond to neighboring $\mathrm{O}$ atoms in ICANAD.

Pb: The covalent radius of $\mathrm{Pb}$ was changed from $1.46 \AA$ to $1.90 \AA$, in order for $\mathrm{Pb}$ to bond to neighboring $\mathrm{O}$ and $\mathrm{N}$ atoms in UDEPIE.

Mo: The covalent radius of Mo was changed from $1.54 \AA$ to $1.57 \AA$, in order for Mo to bond to $\mathrm{O}$ and $\mathrm{N}$ atoms in BOJCIN. 
Sn: The covalent radius of $\mathrm{Sn}$ was changed from $1.39 \AA$ to $1.48 \AA$, in order for Sn to bond to $\mathrm{O}$ atoms in QADBII.

Na: The covalent radius of $\mathrm{Na}$ was changed from $1.66 \AA$ to $1.72 \AA$, in order for $\mathrm{Na}$ to bond to $\mathrm{O}$ atoms in SOWYIM.

Zn: The covalent radius of Zn was changed from $1.22 \AA$ to $1.48 \AA$, in order for Zn to bond to $\mathrm{O}$ atoms in MOYNEU.

Ni: The covalent radius of Ni was changed from $1.24 \AA$ to $1.37 \AA$, in order for Ni to bond to $\mathrm{O}$ atoms in VEGXUC.

Cr: The covalent radius of $\mathrm{Cr}$ was changed from $1.39 \AA$ to $1.41 \AA$, in order for Ni to bond to $\mathrm{N}$ atoms in SOJDEB.

Cu: The covalent radius of $\mathrm{Cu}$ was changed from $1.32 \AA$ to $1.46 \AA$, in order for $\mathrm{Cu}$ to bond to $\mathrm{O}$ atoms in $\mathrm{BACMOH.}$

\section{S3 Manual Removal of MOFs}

During the inspection of the graphs created from the MOFs, multiple MOFs stood out because their crystal graphs were disconnected. Further inspection revealed that many of these MOFs represented an interpenetrated structure, while others were missing vital atoms from the original Cambridge Structural Database (CSD) entry. To separate the invalid structures from the interpenetrated ones, the unit cell was replicated once in each dimension, the graph creation was applied to the super cell and the number of connected components was compared to the number from the original unit cell. For a valid structure, the number of components in the graphs should not change when the unit cell is replicated, except for those structures with counterions to keep the structure charge neutral. 
We manually inspected the structures with a growing number of components and removed the following MOFs (reported as CSD reference codes) from consideration: BEZSIK, SOWYOS, XAPSOY, KIVDUP, GETXEK, KIVFAX, XAPYAO, MIZJUB and FEWTUY. Furthermore, if the graph contains over-bonded hydrogen (with more than one bond) or

carbon (with more than four bonds), or if the graph contains an unconnected node (atom), the graph was dismissed.

\section{S4 Gated Recurrent Unit (GRU)}

A gated recurrent unit $(\mathrm{GRU})^{3}$ takes as inputs the hidden representation from the previous time step $\mathbf{h}^{(t)}$, and the message (the new input) $\mathbf{m}^{(t+1)}$, and generates $\mathbf{h}^{(t+1)}$ as follows.

$$
\begin{aligned}
\mathbf{r} & =\sigma\left(\mathbf{W}_{r} \mathbf{m}^{(t+1)}+\mathbf{U}_{r} \mathbf{h}^{(t)}\right) \\
\mathbf{z} & =\sigma\left(\mathbf{W}_{z} \mathbf{m}^{(t+1)}+\mathbf{U}_{z} \mathbf{h}^{(t)}\right) \\
\tilde{\mathbf{h}}^{(t+1)} & =\tanh \left(\mathbf{W} \mathbf{m}^{(t+1)}+\mathbf{r} *\left(\mathbf{U} \mathbf{h}^{(t)}\right)\right) \\
\mathbf{h}^{(t+1)} & =(1-\mathbf{z}) * \tilde{\mathbf{h}}^{(t+1)}+\mathbf{z} * \mathbf{h}^{(t)}
\end{aligned}
$$

where $\sigma$ is the sigmoid activation function, and $*$ is the Hadamard product. The matrices $\mathbf{W}_{r}, \mathbf{W}_{z}, \mathbf{U}_{r}, \mathbf{U}_{z}, \mathbf{W}, \mathbf{U}$ are learned. The vectors $\mathbf{r}$ and $\mathbf{z}$ are reset gate and update gate, respectively, which decide what information should be passed to the output. Specifically, the reset gate decides how much past information to forget; whereas, the update gate helps to determine what new information to add to the hidden representation. This mechanism allows the GRU to filter and selectively store information. A GRU cell can keep information from long ago and remove irrelevant information. 


\section{S5 Correction for Charge Neutrality via Maximum Like- lihood Estimation}

Here we derive eqn. 11 by maximizing the $\log$-likelihood $\mathcal{L}$ of observing charges $\hat{\mathbf{q}}$ on a given MOF:

$$
\mathcal{L}(\hat{\mathbf{q}})=\sum_{v=1}^{n}\left(\log \left(\frac{1}{\sigma_{v} \sqrt{2 \pi}}\right)-\frac{\left(\hat{q}_{v}-\boldsymbol{\varphi}_{v}\right)^{2}}{2 \sigma_{v}^{2}}\right)
$$

subject to the charge neutral constraint $\sum_{v=1}^{n} \hat{q}_{v}=0$. We can ignore the first term as it does not contain $\hat{\mathbf{q}}$ and change our objective into:

$$
\operatorname{minimize} \sum_{v=1}^{n} \frac{\left(\hat{q}_{v}-\boldsymbol{q}_{v}\right)^{2}}{2 \sigma_{v}^{2}}, \text { subject to: } \sum_{v=1}^{n} \hat{q}_{v}=0
$$

We construct the Lagrangian function with $\lambda$ as the Lagrange multiplier:

$$
l(\hat{\mathbf{q}} ; \lambda)=\sum_{v=1}^{n} \frac{\left(\hat{q}_{v}-\boldsymbol{q}_{v}\right)^{2}}{2 \sigma_{v}^{2}}+\lambda \sum_{v=1}^{n} \hat{q}_{v}
$$

Now

$$
\begin{gathered}
\frac{\partial l(\hat{\mathbf{q}})}{\partial \hat{q}_{v}}=\frac{\hat{q}_{v}-\boldsymbol{q}_{v}}{\sigma_{v}^{2}}+\lambda=0 \\
\Longrightarrow \hat{q}_{v}=\vartheta_{v}-\lambda \sigma_{v}^{2}
\end{gathered}
$$

Plugging this into the charge neutrality constraint, we have:

$$
\begin{gathered}
\sum_{v=1}^{n}\left(\AA_{v}-\lambda \sigma_{v}^{2}\right)=0 \\
\Longrightarrow \lambda=\frac{\sum_{v=1}^{n} \phi_{v}}{\sum_{v=1}^{n} \sigma_{v}^{2}}
\end{gathered}
$$

Substituting this in Equation S6

$$
\hat{q}_{v}=\varphi_{v}-\frac{\sigma_{v}^{2}}{\sum_{u=1}^{n} \sigma_{u}^{2}} \sum_{u=1}^{n} \varphi_{u}
$$




\section{S6 Hyperparameters and Training}

The dimensions of the element embedding, $r$, dimensions of the hidden node features, $k$, and number of message passing time steps, $T$, are hyper-parameters of our MPNN. We investigate the effect of these hyperparameters as shown in Figure S2. The effects of changing $T$ are significant as compared to $r$ and $k$. We select $r=10, k=30$, and $T=4$.

Table S1: Table of hyper-parameters we used for our MPNN.

\begin{tabular}{lll} 
symbol & description & value \\
\hline$r$ & size of element embeddings & 10 \\
$k$ & size of hidden node representations and message & 30 \\
$T$ & number of message passing time steps & 4
\end{tabular}

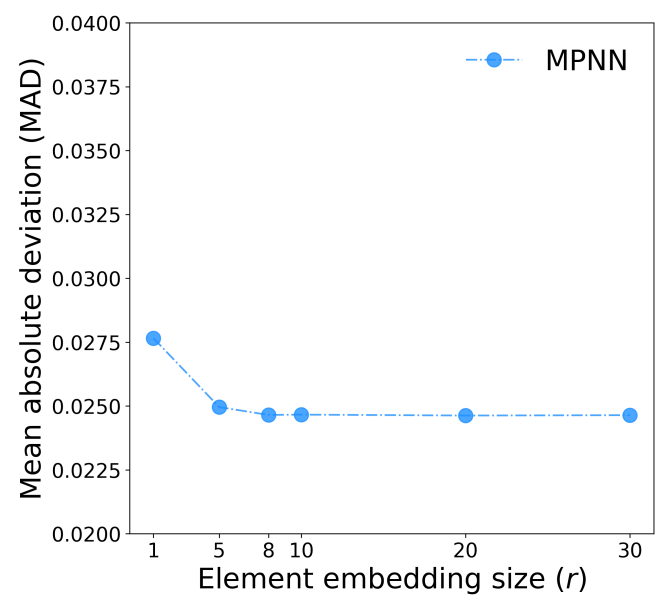

(a)

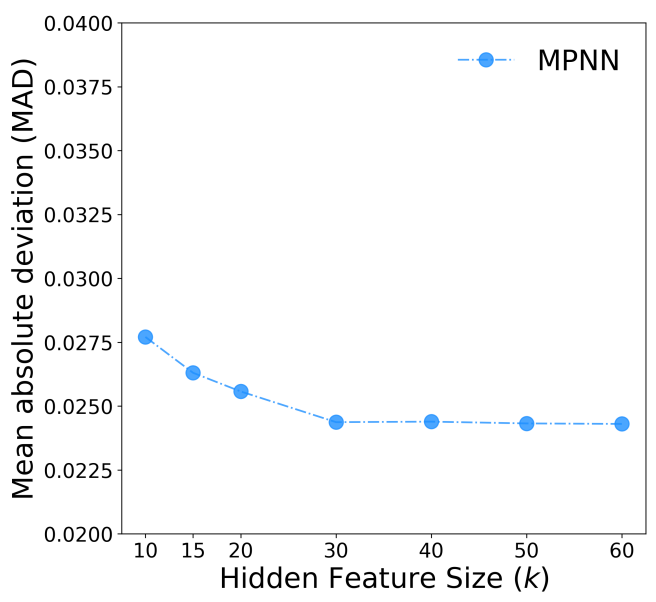

(b)

Figure S2: The effects of changing (a) the size of the element embedding, $r$, while holding $k=30$ fixed (b) the size of the hidden node features, $k$, while holding $r=10$ fixed. $T=4$ is fixed also.

In each training session, we split the dataset into training, validation, and test sets randomly. We ensure that training set contains all element types. Loss is calculated on training set and propagated back to train the model. Validation set is used to make sure model is not overfitted and model with best validation loss is selected. Test dataset is utilized to evaluate the performance of the model. One training session is shown in Figure S3. 


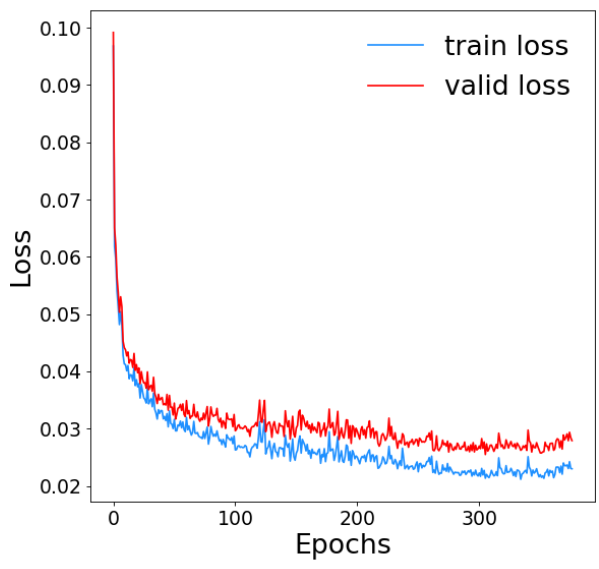

Figure S3: An example session of training the MPNN: the loss on the training (blue) and test (red) set as the number of stochastic gradient descent epochs increases.

\section{S7 Uniform Distribution of Excess Charge}

In a simpler approach to enforce charge neutrality, we instead, after the end of the message passing phase, use a fully connected neuron with shared weights $\boldsymbol{\beta}_{\varphi}$ across all nodes to directly output a before-constraint predicted charge ${ }_{v}$ on each node from the learned node representation:

$$
१_{v}=\boldsymbol{\beta}_{Q}^{\top}\left[\mathbf{h}_{v}^{(L)}, 1\right]
$$

Because we are predicting the charge on each atom independently (but conditioned on its local environment), the molecule is likely not charge-neutral, i.e. $\sum_{v=1}^{n} \Upsilon_{v} \neq 0$, hence "beforeconstraint predicted charge". To enforce charge neutrality, we now uniformly distribute the negative of the net before-constraint charge on the MOF among its atoms to arrive at the final predicted charges:

$$
\hat{q}_{v}=\mathrm{Q}_{v}-\frac{1}{n} \sum_{u=1}^{n} \mathrm{Q}_{u} .
$$

While this approach enforces the charge neutrality constraint in eqn. 2, it does not account for the variance in charge among atoms in a given local environment: more (less) slack should be given to atoms in a context exhibiting a high (low) variance in charge. 


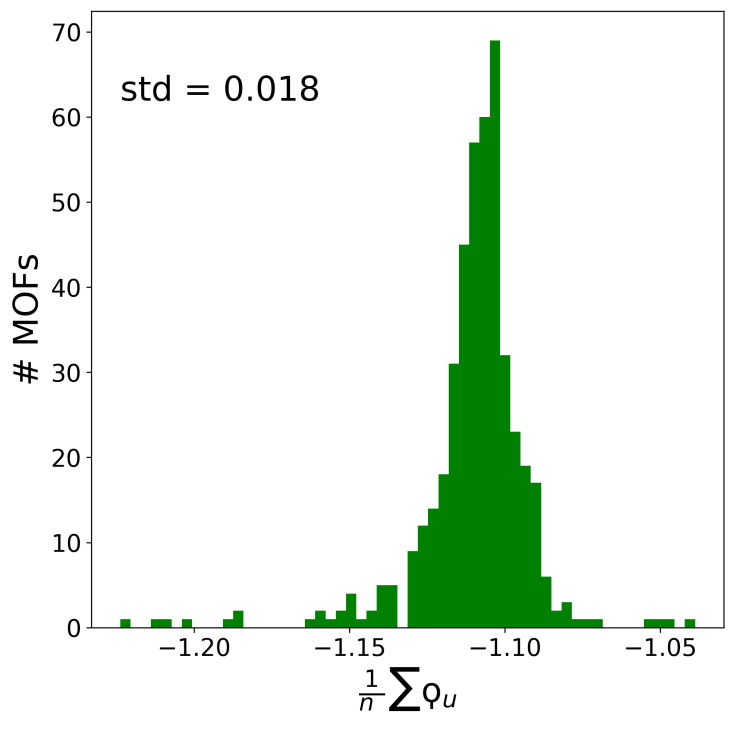

(a) uniform correction

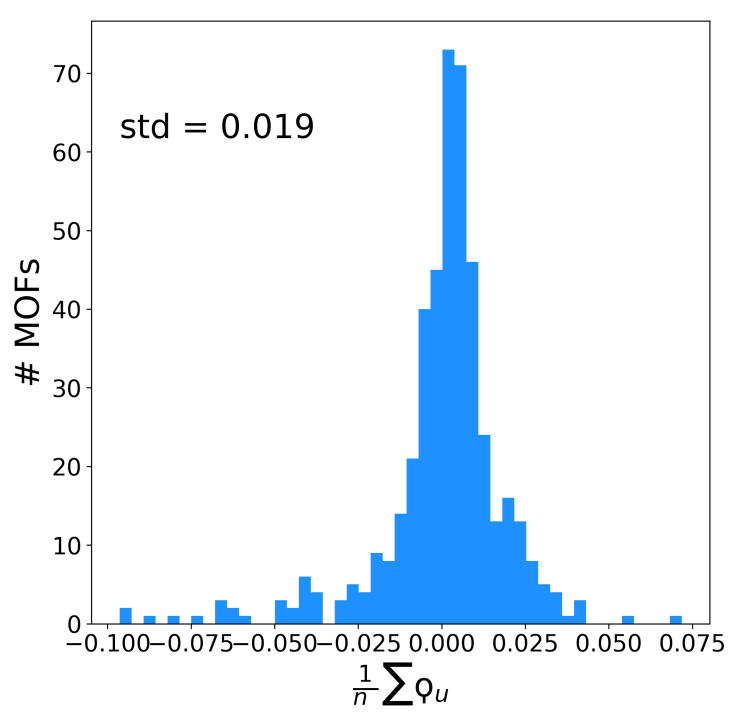

(b) gaussian correction

Figure S4: A histogram of the before-constraint excess charge per atom, $\frac{1}{n} \sum_{u}{ }{ }_{u}$, among the MOFs using two different strategies for predicting before-constraint charges $\varphi_{v}$ 's: (a) eqn. S8 with uniform distribution of excess charge (b) eqn. 8 with variance-based distribution of excess charge. Note that the histograms are not centered at zero; this is because both MPNNs can learn the typical charge on an atom within a given local atomic environment, shifted by a constant, and the charge correction layer will cancel the constant. The width of the histogram (a metric of which is the standard deviation (std)) is meaningful, however, because it describes the variance in excess preliminary charge per atom that is distributed among the MOF atoms to enforce charge neutrality. 


\section{S8 Interpreting the Elemental Embedding}

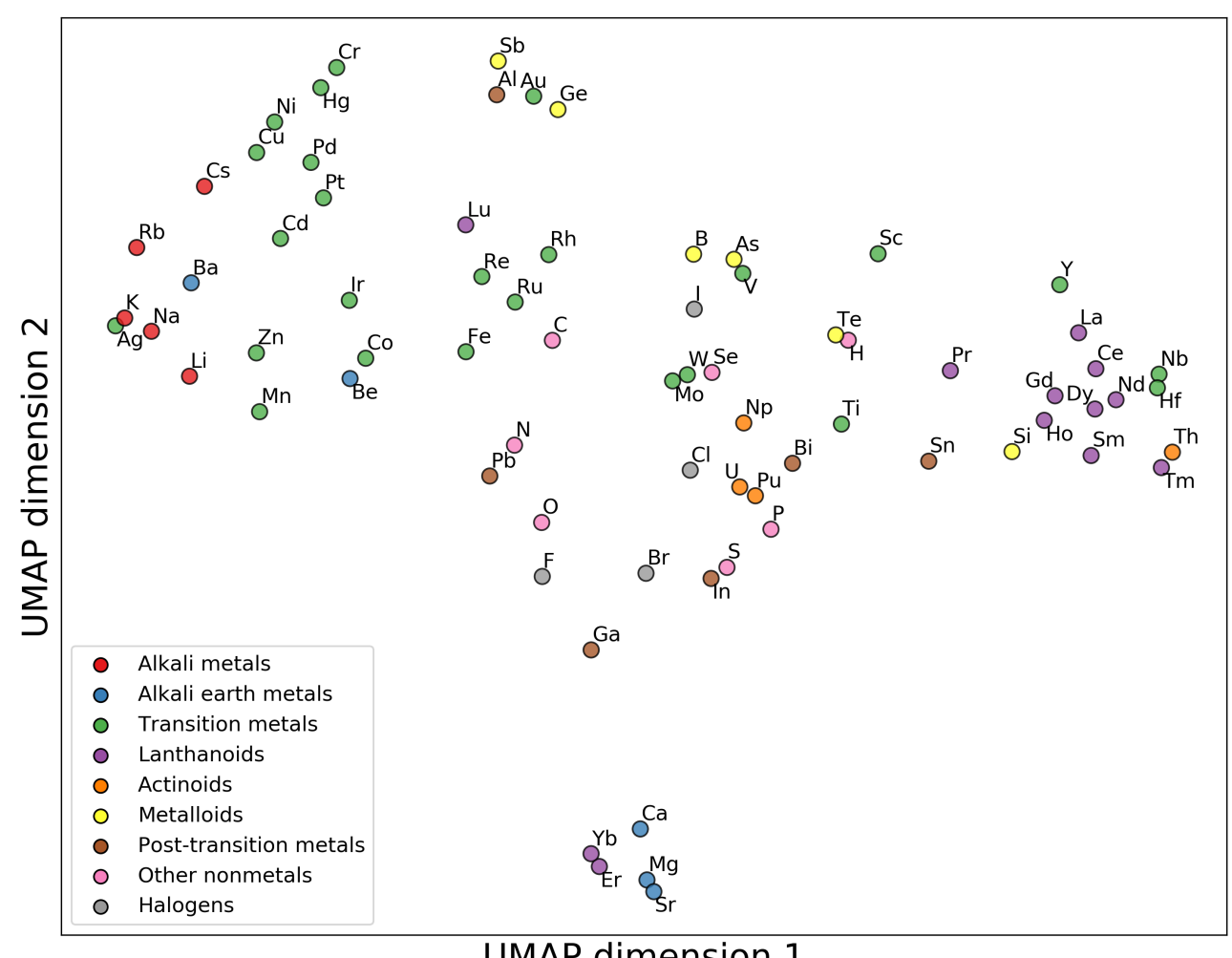

UMAP dimension 1

Figure S5: A 2-dimensional visualization of the element embedding of the MPNN. The embedding originally has $r=10$ dimensions and was reduced to two using $\mathrm{UMAP}^{4}$ (number of neighbors chosen as 8 with a minimum distance of 0.05 ). Each element is colored by its respective periodic table family. 


\section{S9 Element Types in Deployment}

As shown in Fig. S1, some chemical elements are contained in only one MOF. When we deploy our MPNN model, we refrain from predicting the charge on atoms of these chemical elements because the MPNN likely cannot accurately predict them owing to the scarcity of training examples. We choose to keep the MOF structures containing such elements in our training set, with the justification that we can learn about charges on other atoms from these MOFs. Our trained MPNN model for deployment can predict charges for elements shown in Table S6.

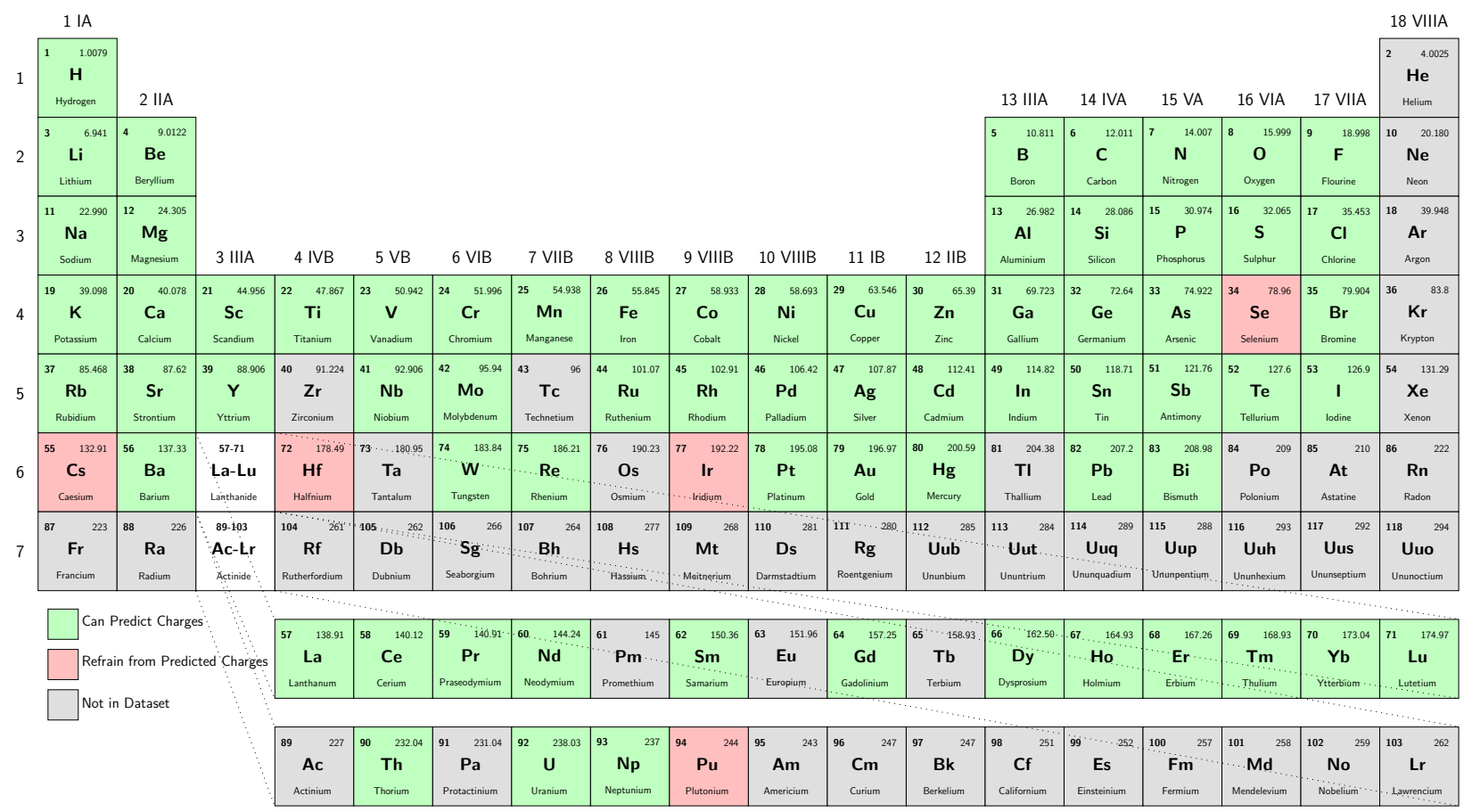

Figure S6: A list of the elements the MPNN model is able to predict partial charges for (colored green). Elements colored gray are elements that never occur in the DFT-derived DDEC charge-assigned MOFs. ${ }^{1}$ The elements we refrain from predicting charges for ( $\{\mathrm{Se}$, Fr, Hf, Ir, Pu\} (colored red) only occur in one DFT-derived DDEC-charge-assigned MOF (each). 


\section{S10 Distribution of predicted variance grouped by el- ement}

Fig. S7 shows the distribution of the predicted variance by our MPNN, $\sigma_{v}^{2}$, grouped by element type.

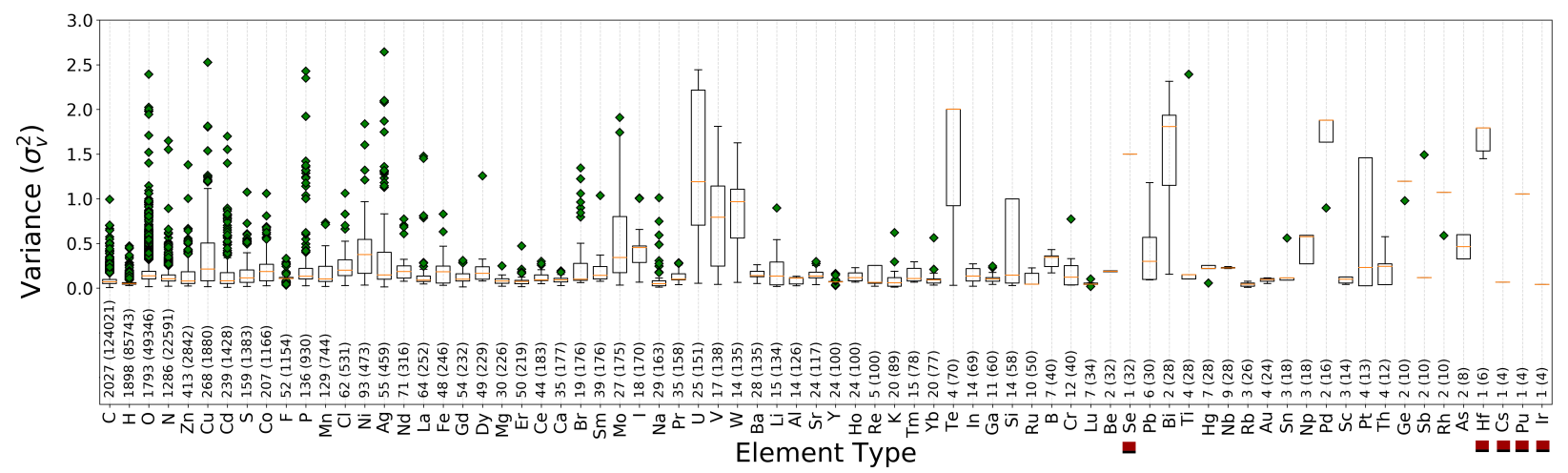

Figure S7: The distribution of the variance predicted by the MPNN, $\sigma_{v}^{2}$, grouped by element type. We trained our model on $90 \%$ of the entire data set (10\% is used for validation). The numbers for each element in the bottom of the figure, $X(Y)$, show $X=$ the number of MOFs containing that element type, $Y=$ the total instances of that element type in all MOFs. Rare element types that are contained only in one MOF are marked with red squares. 


\section{S11 Investigation into outlier in parity plot}

The parity plot in Fig. 4 shows the predicted MPNN charges in the test set of MOFs against the corresponding DFT-derived, DDEC charges. A noticeable outlier is in the center right side of the graph (roughly 2.8 DDEC charge and 0.9 predicted charge), which we here investigate.

The outlying charge is a $\mathrm{Cu}$ atom in $\mathrm{XAFNOH}_{-}$clean.cif.

To understand this discrepancy, we gathered the partial charges of all $\mathrm{Cu}$ atoms in the DFT-derived, DDEC charge-assigned MOFs and visualized the distribution in Fig. S8. The right-most bin in Fig. S8 contains the partial charge on the $\mathrm{Cu}$ atom in XAFNOH_clean.cif (marked). As Fig. S8 depicts, the $\mathrm{Cu}$ atom in XAFNOH_clean.cif is a clear outlier in the DFT-derived, DDEC-charge assigned MOFs. Our MPNN model (incorrectly) assigns a partial charge to the $\mathrm{Cu}$ atom in $\mathrm{XAFNOH}_{-} \mathrm{Clean}$. cif consistent with the typical charge of a $\mathrm{Cu}$ atom in the DFT-derived, DDEC charge-assigned MOFs. This is expected behavior of a supervised machine learning algorithm. Thus, we have explained the outlier in Fig. S8 not necessarily as a problem with our MPNN, but rather as an outlier among $\mathrm{Cu}$ atoms in the original DFT-derived, DDEC charge-assigned MOFs. 


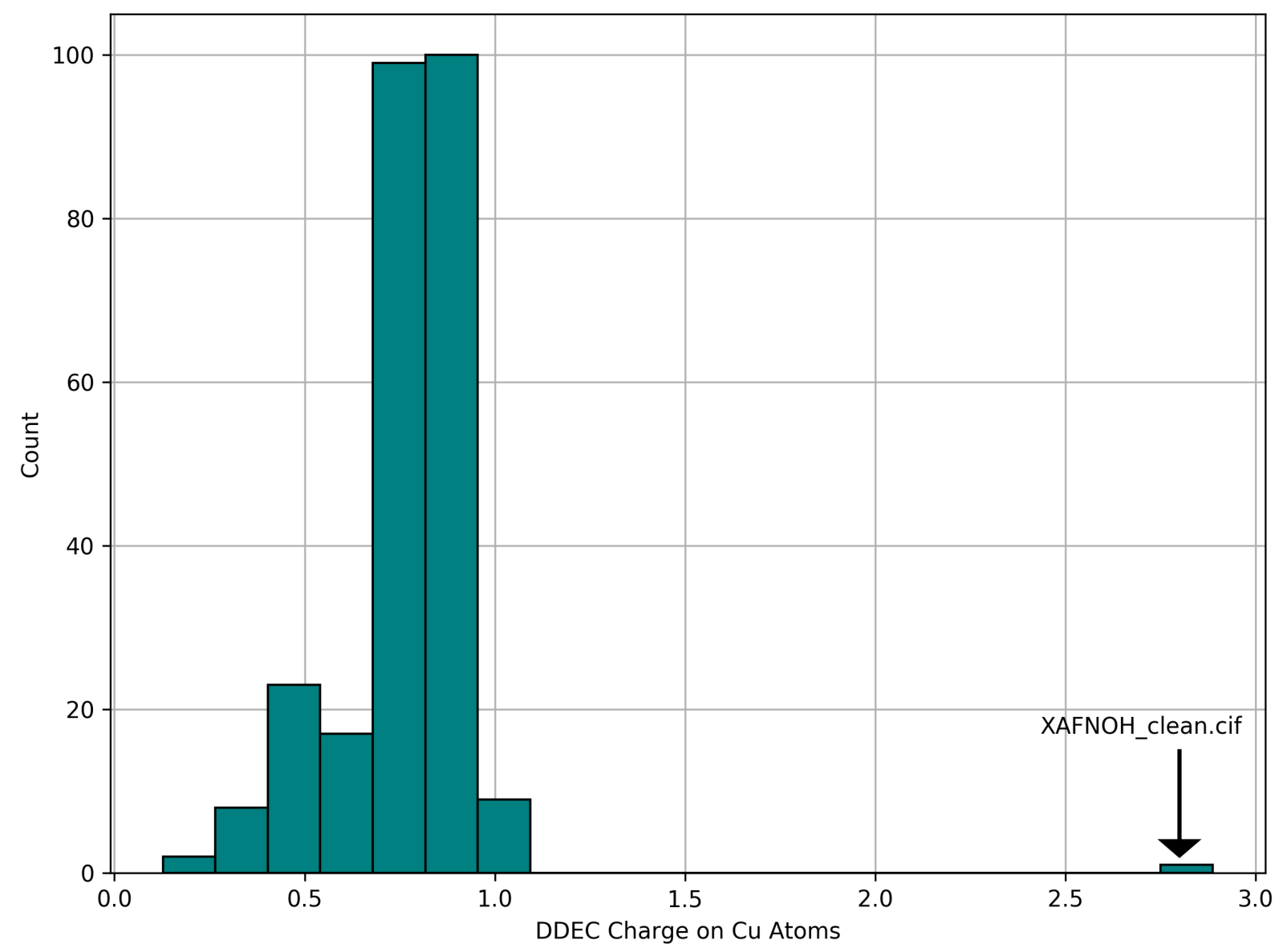

Figure S8: The distribution of (unique) $\mathrm{Cu}$ charges in the DFT-derived DDEC chargeassigned MOFs. We merged identical charges (i.e., we look at the unique $\mathrm{Cu}$ charges), stemming from symmetrically equivalent $\mathrm{Cu}$ atoms within a $\mathrm{MOF}$, to get a better depiction of the distribution: owing to symmetry, the same atomic environment is often replicated multiple times in the same MOF. The distribution is roughly centered around 0.8 , with the $\mathrm{Cu}$ atom in $\mathrm{XAFNOH}_{-} \mathrm{Clean}$.cif an outlier, sitting alone in the right-most bin. 


\section{S12 Runtime performance benchmark}

To characterize the runtime of MOF charge assignment by our MPNN model, we used BenchmarkingTools. $j 1$ in the Julia programming language. We randomly selected $50 \mathrm{MOFs}$ from the database of DFT-derived, DDEC charge-assigned MOFs, then noted the runtime to assign new MPNN charges to them using the Docker image available on our accompanying Github repository. We used an Intel ${ }^{\circledR}$ Xeon ${ }^{\circledR}$ CPU E3-1230 v5 processor.

The runtime includes reading in the .cif file, inferring the bonds between atoms of the structure (i.e., constructing the crystal graph), running the crystal graph (with node features encoding the atomic identities) through the trained MPNN model, and writing the new MPNN-charge-assigned MOF structure to a .cif file. We did not include the precompilation of the involved Julia packages in the runtimes, since, once compiled, an arbitrary of MOFs can be passed to the (compiled) Julia functions. To statistically ensure valid runtimes, BenchmarkingTools.jl assigned charges to each MOF multiple times; we report the mean of these runtimes here.

The expected runtime of MPNN-charge assignment to a MOF is $\sim 3$ seconds.

The runtime of MPNN-charge assignment scales with number of atoms in the MOF. To capture that trend, Fig. S9 shows the relationship between the runtime and the number of atoms in the MOFs. 


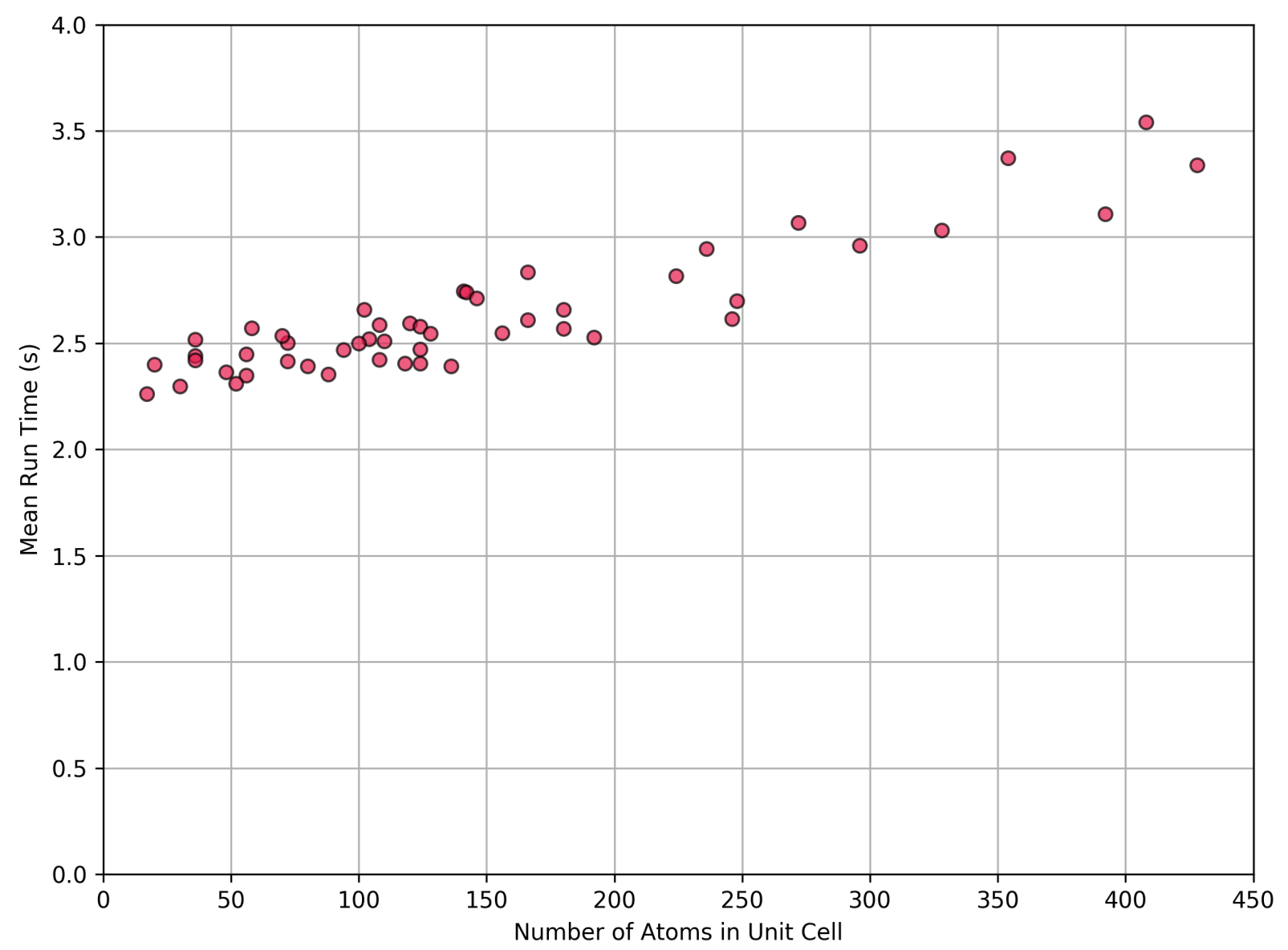

Figure S9: The mean run time of MPNN charge assignment for 50 different MOFs and its relationship to the number of atoms in the MOF. 


\section{S13 Molecular simulations of carbon dioxide adsorp- tion}

To further judge the quality of the MOF charges assigned by our MPNN model, we computed the Henry coefficient of carbon dioxide in 171 MOFs at $298 \mathrm{~K}$ using Widom insertions, ${ }^{5}$ with MOF charges assigned using four different schemes: (1) MPNN charges, (2) DFT-derived, DDEC charges, ${ }^{1}$ (3) I-QEq charges, ${ }^{6}$ and (4) zero charges $\left(q_{v}=0 \forall v \in \mathcal{V}\right)$. Our objective is to compare the Henry coefficients of carbon dioxide in the MOFs with the highest fidelity charges, DFT-derived, DDEC charges ${ }^{1}$ to the Henry coefficients in MOFs under the other charge assignment schemes (MPNN, I-QEq, no charges). We include in this investigation only MOFs that belong to the test set from the MPNN training session to ensure we are assessing generalization error. We did not use every MOF from the test set, as we were only able assign I-QEq charges to 212 MOFs - due to e.g., missing atom ion energy data or due to the solution not converging - (leaving 242 without I-QEq charge assignment) and, out of the remaining 212 MOFs, 41 ran into other errors during the simulation, such as cores running out of memory or jobs not completing after a substantial amount of time. The code we used to assign the I-QEq charges was obtained from the original I-QEq paper by Wells et al. ${ }^{6}$ This code uses atom ionization energy parameters computed with the def2 basis set, as opposed to the modified def2+exp parameters used by Ongari et al. ${ }^{7}$ For our force field, we use the TraPPE model ${ }^{8}$ for $\mathrm{CO}_{2}$ and the Universal Force Field (UFF) ${ }^{9}$ for the MOF structures. Lorentz-Berthelot mixing rules give the Lennard-Jones parameters for the cross-species interactions. We impose a $12.5 \AA$ cutoff radius, beyond which we neglect Van der Waals interactions. Importantly, the TraPPE $\mathrm{CO}_{2}$ model places partial point charges on the atoms of $\mathrm{CO}_{2}$ to model electrostatic interactions; therefore, this is an indirect test of the MPNN charges to adequately describe the electrostatic potential in the MOF, in comparison to DFT-derived, DDEC charges. To estimate the Henry coefficient in each MOF, we used 1000 Widom insertions per unit volume $\left[\AA^{3}\right]$ of the simulation supercell. The 
unit cell of each MOF was replicated in each dimension such that a $12.5 \AA$ sphere would fit into the newly created supercell. We emphasize that the simulation settings for the Henry coefficient are identical under all charge assignment schemes in this investigation (with the exception of the charges assigned to the MOF atoms). We use the open-source Julia package, PorousMaterials.jl, to carry out the simulations.

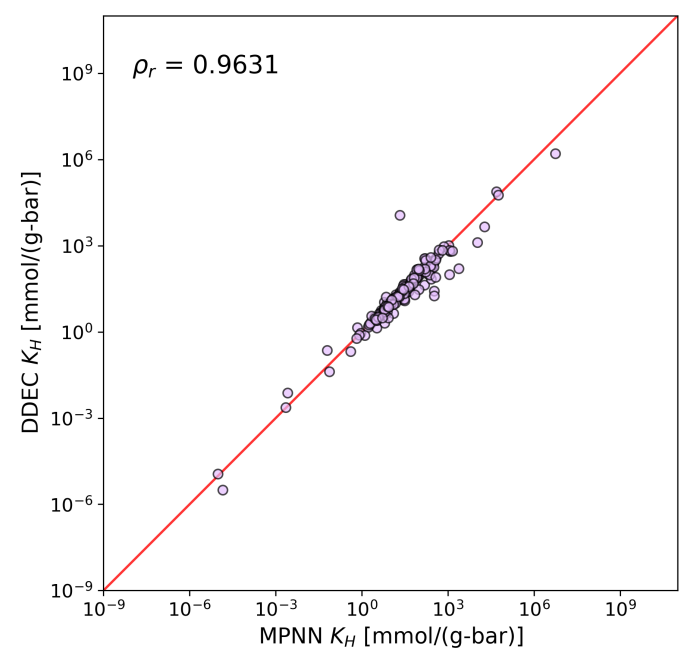

(a)

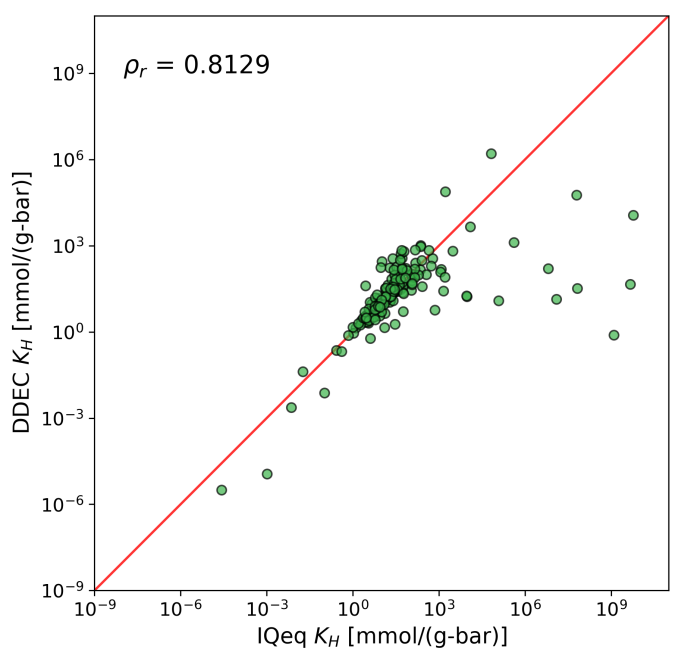

(b)

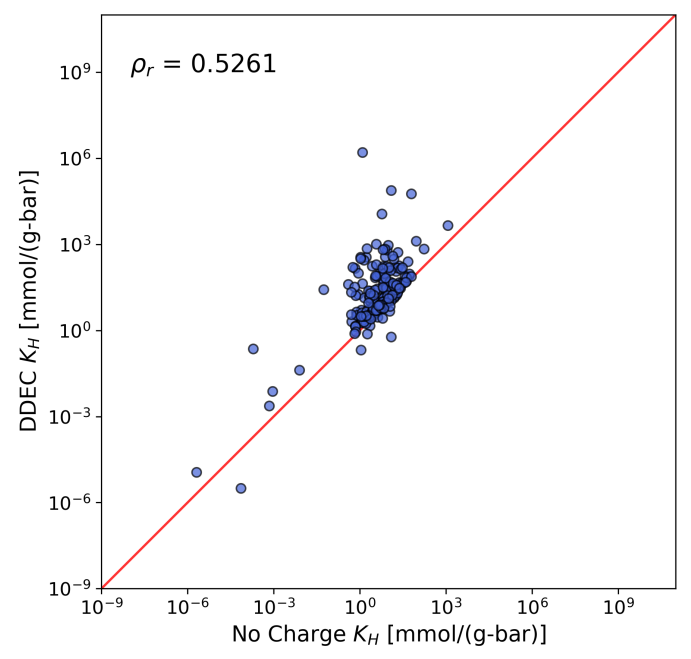

(c)

Figure S10: Parity plots of $\mathrm{CO}_{2}$ Henry coefficients, $K_{H},(298 \mathrm{~K})$ computed in MOFs with DFT-derived, DDEC charges $^{1}$ against those computed in MOFs with (a) MPNN charges, (b) I-QEq charges, and (c) zero charges. Each point in this plot represents a MOF structure. Diagonal line denotes equality. Spearman's rank correlation coefficient is reported on each figure as $\rho_{r}$. 
The parity plot in Fig. S10 shows the relationship between the $\mathrm{CO}_{2}$ Henry coefficients in MOFs with high-fidelity DDEC charges against those in the same MOFs with MPNN (a), I-QEq (b), and zero charges (c). Each point in this plot represents a MOF. We use Spearman's rank correlation coefficient, $\rho_{r}$, between Henry coefficients as a metric to compare the Henry coefficients under different charge assignment schemes. The Henry coefficients in MPNN-charge-assigned MOFs (1) exhibit good agreement with the Henry coefficients in the DFT-derived, DDEC charge-assigned MOFs and $(2)$ are more correlated $\left(\rho_{r}=0.96\right)$ with those of the MOFs with DFT-derived, DDEC-charges than when I-QEq is used ( $\rho_{r}=$ 0.81). Moreover, emphasizing the importance of accurate charge assignment for predicting adsorption in MOFs via simulation, the Henry coefficients in MOFs without charges were poorly correlated $\left(\rho_{r}=0.52\right)$ with those in the DFT-derived, DDEC-charge-assigned MOFs. Notably, the contribution of electrostatic interactions to simulated adsorption varies among adsorbates (well, the molecular models for the adsorbates). 


\section{References}

(1) Nazarian, D.; Camp, J. S.; Sholl, D. S. A comprehensive set of high-quality point charges for simulations of metal-organic frameworks. Chemistry of Materials 2016, 28, 785-793.

(2) Cordero, B.; Gómez, V.; Platero-Prats, A. E.; Revés, M.; Echeverría, J.; Cremades, E.; Barragán, F.; Alvarez, S. Covalent radii revisited. Dalton Transactions 2008, 2832-2838.

(3) Cho, K.; Van Merriënboer, B.; Gulcehre, C.; Bahdanau, D.; Bougares, F.; Schwenk, H.; Bengio, Y. Learning phrase representations using RNN encoder-decoder for statistical machine translation. arXiv preprint arXiv:1406.1078 2014,

(4) McInnes, L.; Healy, J.; Melville, J. Umap: Uniform manifold approximation and projection for dimension reduction. arXiv preprint arXiv:1802.03426 2018,

(5) Frenkel, D.; Smit, B. Understanding molecular simulation: from algorithms to applications; Elsevier, 2001; Vol. 1.

(6) Wells, B. A.; De Bruin-Dickason, C.; Chaffee, A. L. Charge equilibration based on atomic ionization in metal-organic frameworks. The Journal of Physical Chemistry C 2015, $119,456-466$.

(7) Ongari, D.; Boyd, P. G.; Kadioglu, O.; Mace, A. K.; Keskin, S.; Smit, B. Evaluating charge equilibration methods to generate electrostatic fields in nanoporous materials. Journal of Chemical Theory and Computation 2018, 15, 382-401.

(8) Potoff, J. J.; Siepmann, J. I. Vapor-liquid equilibria of mixtures containing alkanes, carbon dioxide, and nitrogen. AIChE Journal 2001, 47, 1676-1682.

(9) Rappé, A. K.; Casewit, C. J.; Colwell, K.; Goddard III, W. A.; Skiff, W. M. UFF, a full periodic table force field for molecular mechanics and molecular dynamics simulations. Journal of the American chemical society 1992, 114, 10024-10035. 